ARTICLE

DOI: $10.1038 /$ ncomms 7912

OPEN

\title{
Superlattices assembled through shape-induced directional binding
}

Fang Lu ${ }^{1}$ Kevin G. Yager ${ }^{1}$, Yugang Zhang ${ }^{1}$, Huolin Xin ${ }^{1} \&$ Oleg Gang ${ }^{1}$

Organization of spherical particles into lattices is typically driven by packing considerations. Although the addition of directional binding can significantly broaden structural diversity, nanoscale implementation remains challenging. Here we investigate the assembly of clusters and lattices in which anisotropic polyhedral blocks coordinate isotropic spherical nanoparticles via shape-induced directional interactions facilitated by DNA recognition. We show that these polyhedral blocks-cubes and octahedrons-when mixed with spheres, promote the assembly of clusters with architecture determined by polyhedron symmetry. Moreover, three-dimensional binary superlattices are formed when DNA shells accommodate the shape disparity between nanoparticle interfaces. The crystallographic symmetry of assembled lattices is determined by the spatial symmetry of the block's facets, while structural order depends on DNA-tuned interactions and particle size ratio. The presented lattice assembly strategy, exploiting shape for defining the global structure and DNA-mediation locally, opens novel possibilities for by-design fabrication of binary lattices.

\footnotetext{
${ }^{1}$ Center for Functional Nanomaterials, Brookhaven National Laboratory, Upton, New York 11973, USA. Correspondence and requests for materials should be addressed to O.G. (email: ogang@bnl.gov).
} 
C ontrolling the organization of nanoparticles in superlattices is important for creating materials with emergent and collective properties for applications in photonics, catalysis, biomaterials and high-energy composites ${ }^{1-3}$. Spherical particles generally assemble into lattice symmetries determined by simple packing criteria. Richer phase behaviour has been observed by exploiting more sophisticated interaction potentials; for instance, exploiting ligand and DNA shells ${ }^{3-8}$, van der Waals ${ }^{9}$, electrostatic ${ }^{10,11}$, dipolar ${ }^{12,13}$ and magnetic forces ${ }^{11}$. Although much diversity has been demonstrated, the highly system-dependent results point towards difficulties for the predictable fabrication of designed materials.

An alternative paradigm is to introduce directional bonds between spherical particles, thereby coordinating species in the particle's local environment based on the bonding geometry rather than packing. One possible realization is 'patchy particles ${ }^{12,14-16}$, where spherical particles are decorated with specifically arranged binding regions ${ }^{17}$. The resultant anisotropic interactions mimic the directional bonds of atomic systems. This strategy has been highly successful at the micron scale, with demonstration of assembling designed clusters ${ }^{17,18}$. However, as shown by theoretical studies $14,19,20$, the formation of ordered extended lattices from such particles might be hindered by local orientational defects that cannot easily heal; experimentally, only one-dimensional (1D) and two-dimensional (2D) organizations of colloids were shown ${ }^{13,18}$. Recent progress ${ }^{21-23}$ in transferring this concept to the assembly of nano-scale systems is promising; however, it still remains comparatively elusive because of the difficulties in large-scale synthesis of nanoparticles with welldefined anisotropic patches. In this work, we present an alternative idea: the ordering of spherical nanoparticles can be dictated through directional bonds provided by DNA-encoded faceted nanoscale 'blocks'. We demonstrate, using cubic and octahedral nanoblocks, that well-defined three-dimensional (3D) lattices of spheres and blocks can be formed within appropriate regimes of nanoparticle sizes and DNA shells.

\section{Results}

Lattices of hetero-shaped particles via directional bonds. In our approach, schematically shown in Fig. 1, bonding directionality is inherently determined by the block's anisotropic shape through attractive facets. As a result, spheres can be coordinated into

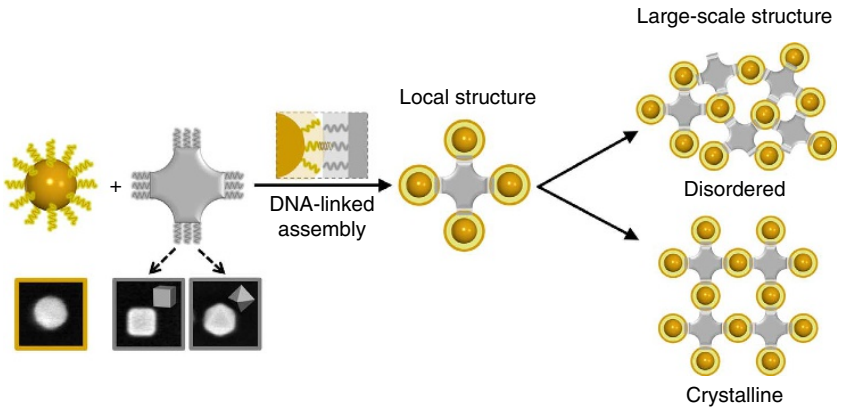

Figure 1 | Schematic of shape-induced directional bonds for DNA-linked nanoparticle assembly. SNPs (yellow unit) are isotropic objects with high symmetry. ANPs (grey unit) have low-order symmetry; for example, CB or $\mathrm{OC}$ as shown in electron micrographs (inner frame length of images is $100 \mathrm{~nm}$ ). Regulated interactions between particles are induced by functionalizing SNPs and ANPs with complementary DNA strands. The directional bonding of ANPs coordinates SNPs in a local structure (clusters). Our study examines the conditions under which the shape of ANPs can be translated into well-established local structure, and factors affecting the formation of either disordered or ordered large-scale 3D assembly. clusters in accordance with the geometry of the shaped object. Ultimately, large-scale binary lattices can be formed in a predictable manner. In this study we realize this concept by employing directional bonds provided through polyhedral nano blocks, either cubes (CBs) or octahedrons (OCs), for assembly of spherical nanoparticles. The mutual attraction of heterogeneously shaped particles is required to suppress phase separation ${ }^{24-26}$. In this regard, we functionalize both shaped particles (spherical and polyhedral) with DNA strands, allowing us to 'encode' particle interactions via DNA sequence. We enforce attractive interactions between different-shape particles via DNA complementary, while same-shape particles are repulsive because of entropic chain effects. In addition, the softness of DNA shell plays an important role in accommodating dissimilar particle curvatures. We stress that in the proposed approach, no complex engineering of spherical particles is required, and this method can take advantage of the wide variety of available nanoscale-shaped objects $^{29}$.

Despite the conceptual simplicity of this approach for generating designed clusters, it is nontrivial to predict the requirements for the formation of extended, ordered $3 \mathrm{D}$ superlattices (Fig. 1). Indeed, the geometrical constraints imposed by shapes $^{26,28,29}$ result in a rich structural diversity even in monocomponent assemblies of shaped objects ${ }^{30-36}$. Thus, it is not yet clear what dictates or inhibits the formation of large-scale ordered arrays even for the simplest case of hetero-shapes: mutually attractive spherical and shaped objects. At a general level, different particle shapes will exhibit significantly different anisotropic interactions in a system, thereby imposing conflicting local constraints that may override intended bonding directionality. Such conflicts can be resolved via complementaryshaped particles due to the favourable packing ${ }^{37-39}$, certain shapes can be accommodated at the right size regimes ${ }^{40}$ or by optimizing a delicate balance of interactions and space-filling requirements ${ }^{11,41}$. In our approach, the use of DNA permits tuning the interaction 'softness' due to DNA's polymeric nature. This allows us to probe the complex interplay between shapeinduced directional binding, the interaction potential and geometrical requirements due to the particle size-ratios. As we show below, ordered assemblies of disparate shapes do not arise generically, even in the presence of well-defined directional bonds. Instead, crystals are obtained only with appropriate selection of interaction potentials and particle size ratio, in particular, those that satisfy local geometric constraints. Thus, directional bonds can be optimized to yield highly ordered binary $3 \mathrm{D}$ superlattices of hetero-shaped nanoparticles, wherein global crystallographic symmetry is directly selected by particle shape.

To explore the proposed strategy (Fig. 1) for assembly of a binary assembly of hetero-shaped particles, we used spherical nanoparticles (SNPs) as isotropic objects with highest-order symmetry and anisotropic nanoparticle (ANP), shown as a generic grey block in the schematic. Specifically, polyhedral nano objects, either a cube (CB) or an octahedron (OC), were employed in this study. These two shapes are mathematically dual to one another: the point symmetry of $\mathrm{CB}$ facets matches that of $\mathrm{OC}$ vertices. By examining these two closely related shapes, we are able to examine the effects of both particle symmetry and geometry on lattice formation. Regulated interactions between particles are induced by functionalizing ANPs and SNPs with complementary DNA strands. We hypothesize that the ability of ANPs to coordinate SNPs is determined by the faces of ANPs, since DNA hybridization is maximized when area between particles with complementary DNA is increased. For example, due to their six square and eight equilateral triangular faces, respectively, the $\mathrm{CB}$ and $\mathrm{OC}$ are expected to locally coordinate SNPs into six- and eightfold symmetries. On other hand, it is not 
clear under what conditions disordered versus ordered structures will form for large-scale SNP/ANP assemblies, even if local coordination is precisely controlled. We conjecture that the shape symmetry of ANPs can be translated under certain conditions into the global lattice type; thus, the symmetry of the lattice is encoded by geometrical properties of the ANP, while DNA is responsible for local connections of hetero-shaped objects. The formed lattice can be seen as a result of the constraining of a high-order-symmetry element, such as sphere, by a low-ordersymmetry anisotropic object.

Assembly of cube and sphere nanoparticles. We first studied the binary system of spherical and polyhedral nanoparticles using gold $(\mathrm{Au})$ nanosized cubes (CBs). The edge length of $\mathrm{Au} \mathrm{CBs}$ and the diameter of SNPs are selected to be similar (46 nm; Fig. $2 \mathrm{a}$ and Supplementary Fig. 1). We coated Au CBs and SNPs with two types of complementary 18-base single-strand (DNA design denoted as 18S) DNAs, $18 \mathrm{~A}$ and $18 \mathrm{~A}^{\prime}$, respectively (Fig. 2a). Each type of DNA contains a 6-base outer recognition region and a 12-base internal spacer region (see Supplementary Table 1). To study local coordination, we mixed SNPs and CBs in a number ratio of 6:1. Using transmission electron microscopy (TEM), we observed the formation of a large cluster population $(\sim 63 \%)$ in which the central CB is surrounded by six SNPs, along with some incomplete clusters containing a smaller number of SNPs.

To reveal the 3D spatial arrangement of SNPs around the $\mathrm{CB}$, we performed a TEM tomographic reconstruction. A series of TEM images from $-72^{\circ}$ to $+15^{\circ}\left(2^{\circ}\right.$ steps $)$ were acquired from a typical cluster containing six of SNPs coordinated around CB. For our model-based reconstruction, we assumed that the centre particle is cubic ( $46 \mathrm{~nm}$ in edge length) and the surrounding particles are spherical $(46 \mathrm{~nm}$ in diameter; Fig. $2 \mathrm{~b}$ and Supplementary Note 1). The reconstruction results (Fig. 2b central panel, and the corresponding simulated projections on the bottom panel) reveal that six SNPs are positioned on the centres of the six flat facets of the CB (Supplementary Movies 1 and 2). These observations indicate that the cubic particle coordinate SNPs in accordance with a valence determined by the symmetry of CB facets. To promote an unrestricted assembly of a large-scale structure, we mixed CBs and SNPs in a number ratio of 1:1. At the initial stages of aggregation at room temperature, finite-sized clusters of various configurations were observed (two left images on Fig. 2c and Supplementary Fig. 2a). Although a broad population of CB-SNP was detected at this stage, we stress that the maximum observed coordination for $\mathrm{CB}$ was 6 , as defined by its facet-induced valence. At the later assembly stages, the merging of clusters occurred (two right images on Fig. 2c and Supplementary Fig. 2b) and larger-scale assemblies (Supplementary Fig. 2c) were eventually formed. The geometrical constraints and local DNA-induced interactions between CBs and SNPs result in a particular arrangement of particles in the growing agglomerate (Fig. 2c and Supplementary Fig. 2). The CBs and SNPs are positioned in predominantly alternating way; however, only short-range order is realized in this arrested state because of local DNA crowding and strong interactions between complementary adhesive DNA shells.

The formation of equilibrium assemblies in DNA-guided systems requires a re-arrangement of DNA bonds formed during

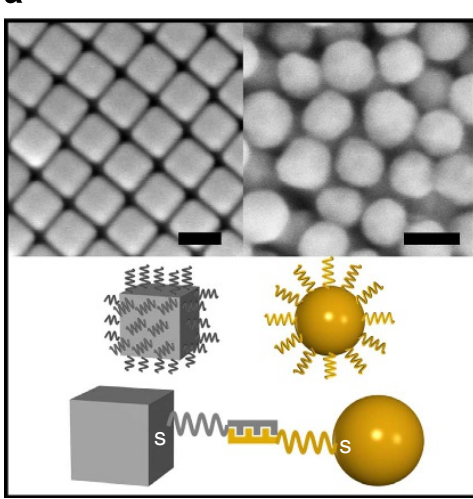

b

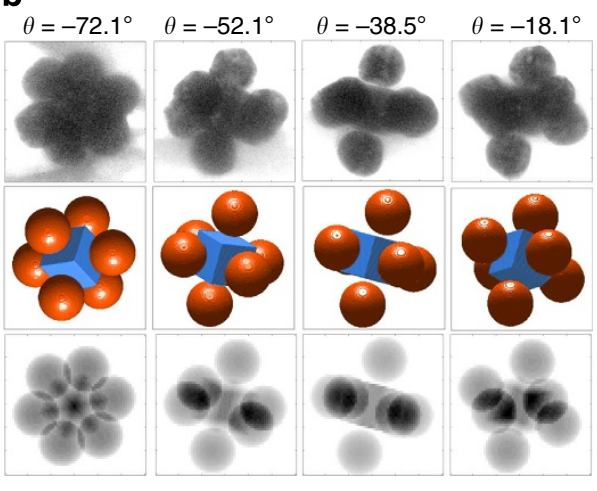

C

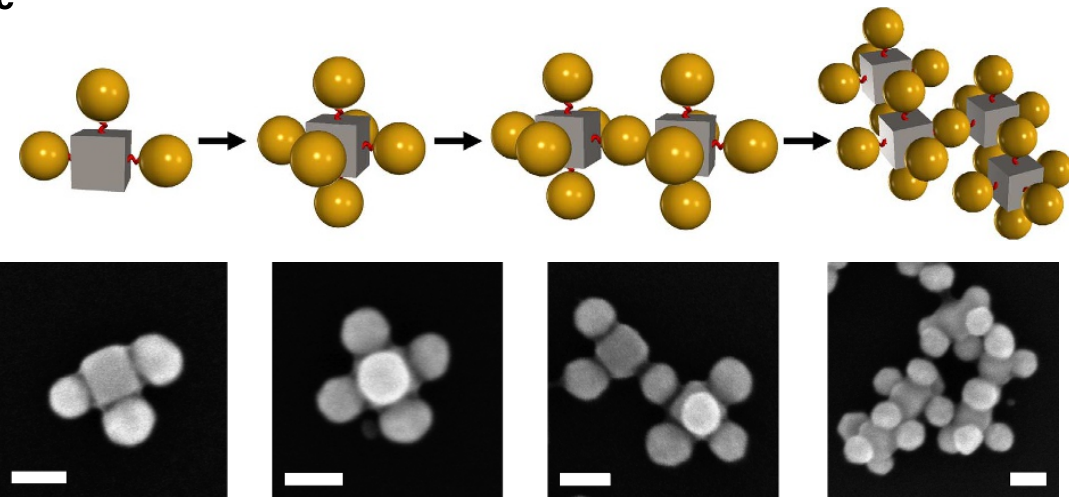

Figure 2 | Cube-encoded assemblies of clusters with spherical nanoparticles. (a; Top) SEM images of (from left to right) CBs (46 nm in edge length) and SNPs ( $46 \mathrm{~nm}$ in diameter); (bottom) schematic of the DNA functionalization and assembly of CB and SNP. (b) Comparison of (top) TEM images with (middle) 3D reconstruction models and (bottom) the projections of the modelled structures at a few selected tilt angles to reveal the 3D structure of the assembled clusters (from left to right: $-72.1^{\circ},-52.1^{\circ},-38.5^{\circ}$ and $-18.1^{\circ}$ ). The projections of the modelled clusters agree with the recorded TEM images. (c; Top) Schematic and (bottom) SEM images illustrate the shape-induced directional bonding of CB in a cluster-dimension-extending history (from left to right). The scale bars, $50 \mathrm{~nm}$. 
the initial uncontrolled aggregation. This transition can be achieved by thermal annealing, which induces partial melting (dissociation) of DNA duplexes, allowing particles to optimize their location in order to maximize the number of DNA hybridizations, that is, to satisfy an energetic criterion. For example, in the SNP/CB system, samples assembled at room temperature were subsequently annealed at a pre-melting temperature $\left(\sim 39^{\circ} \mathrm{C}\right)$ for several hours, followed by gradually cooling back to room temperature. We employed synchrotronbased small-angle X-ray scattering (SAXS) to probe in situ the lattice structure formation and the role of annealing temperature, $T_{\mathrm{a}}$. The peak positions and relative intensities of SAXS peaks provide insights on internal organization of assemblies in solution, while the number of peaks and their widths reflect their degree of ordering. The phase evolution from an amorphous to a crystalline state is observed as $T_{\mathrm{a}}$ approaches the melting temperature gradually (Supplementary Fig. 3). Specifically, very little change is observed until $37^{\circ} \mathrm{C}$; however, at higher temperature, the development of new higher-order peaks in the SAXS pattern, with the corresponding significant sharpening of peaks, indicates a transition to a long-range crystalline ordering.

Indeed, the annealed (at $39^{\circ} \mathrm{C}$ ) lattice exhibits a remarkable degree of order (Fig.3b-c) as evident from the more than 12 sharp peaks in the structure factor, $S(q)$ (Fig.3b, blue line), for the system assembled from 18-base single-strand DNA-functionalized 46-nm CBs and 46-nm SNPs (Fig. 3a). The structure factors were obtained as $S(q)=I_{\mathrm{a}}(q) / I_{\mathrm{p}}(q)$, where $I_{\mathrm{a}}(q)$ and $I_{\mathrm{p}}(q)$ are background-corrected SAXS profiles measured from the aggregate in buffer solution, and free particles in solution, respectively.
The correlation length of the formed superlattice is at least $0.5 \mu \mathrm{m}$, estimated from the width of the first scattering peak as $\xi=2 \pi / \Delta q$, where $\Delta q$ is a resolution-corrected $\left(\Delta q_{\text {res }} \sim 0.0007 \AA^{-1}\right)$ full-width at half maximum, and as confirmed by our detailed analysis (Supplementary Note 2). A quantitative analysis ${ }^{42}$ of $S(q)$, which accounts for lattice type, particle shapes, relative particle orientations and interparticle distances (Supplementary Note 2), provides insight into the structural organization of the lattice formed by CBs and spheres.

The proposed structure, a NaCl-type lattice with space group symmetry of $F m \overline{3} m$, is based on the alternating arrangement of spheres and CBs in the lattice (Fig. 3d), a symmetry imposed by the constraints of 'sticky' cubic ANPs. The modelled $S(q)$ exhibits (Fig. 3b, red line) an excellent agreement with the data (Fig. 3b, blue line), thus confirming that CBs organize SNPs into a facecentred cubic $(f c c)$ lattice with a two-component basis. The position of the first $S(q)$ peak, $q_{1}$, corresponds to the $\{200\}$ crystalline plane in a NaCl-lattice unit cell. There are six nearest SNPs surrounding each CB, and six nearest CB around each SNP, indicating a coordination number of 6 . Such an arrangement of SNP is different from the more conventional body-centred cubic (bcc) lattice formed by the assembly of identical spheres with intercomplementary DNA (Supplementary Fig. 4$)^{3,4}$. On the basis of the analysis (Supplementary Note 3) lattice constant, $a$, for this $\mathrm{NaCl}$-type structure is $120.8 \mathrm{~nm}$, which corresponds to a surfaceto-surface distance between CB and SNP of $d=14.4 \mathrm{~nm}(d=a / 2-$ $L_{\mathrm{CB}} / 2-D_{\mathrm{SNP}} / 2, L_{\mathrm{CB}}$ and $D_{\mathrm{SNP}}$ are the CB edge length and the SNP diameter, respectively). Our analysis indicates that the lattice formed from CBs and SNPs exhibits not only a good translational a

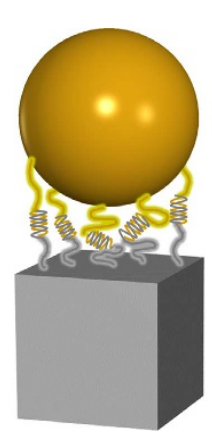

b

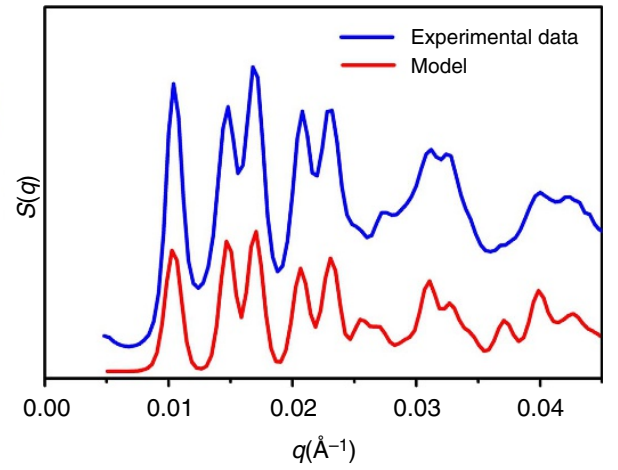

c

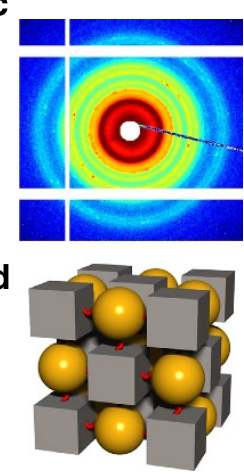

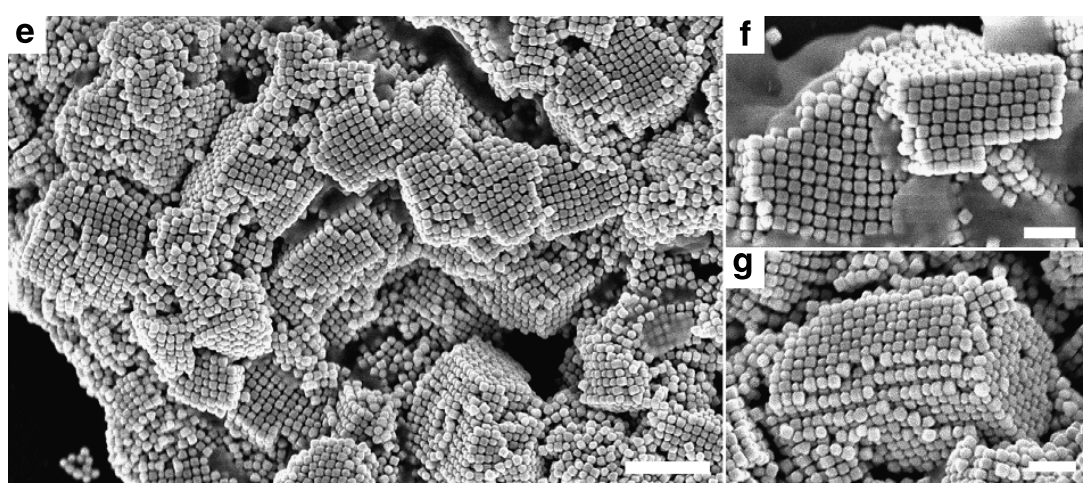

Figure 3 | Cube-sphere nanoparticle assemblies of superlattices. (a) Schematic of a 46-nm SNP/46-nm CB pair linked by DNA system 18S. (b) SAXS data with experimental (blue) and modelled (red) structure factors, $S(q),(\mathbf{c})$ scattering image and (d) the corresponding structure schematic for 46-nm SNP/46-nm CB assembly system, which crystallizes into a NaCl-type lattice with a space group symmetry of Fm $\overline{3} \mathrm{~m}$. (e) Low-magnification SEM image of SNP/CB-assembled crystals, where square-lattice ordering can be observed from the fragments, even though drying effects cause cracks in the crystals (scale bar, $500 \mathrm{~nm}) .(\mathbf{f}, \mathbf{g})$ High-magnification images of superlattice, demonstrating the alternate packing of SNPs and CBs in the 3D square lattice (scale bar, $200 \mathrm{~nm}$ ). 
order, but also that the $\mathrm{CBs}$ are oriented uniformly (Supplementary Note 2). We emphasize that the SAXS measurements are performed in situ, and thus represent the native, solvated configuration of the superlattice. Moreover, the SAXS data demonstrate that only a single phase is formed over the whole macroscopic dimension $(\sim 0.2 \mathrm{~mm})$ of the sample.

To further probe the superlattice structure, particularly to assess the local CB-SNP arrangements, we used direct visualization using scanning electron microscopy (SEM) after the annealed aggregates were deposited on a substrate. A lowmagnification image (Fig. 3e) shows that, although drying unavoidably induces breaks in the crystallites, the long-range crystalline order is clearly evident within micron-sized domains. The domains visualized after drying in fact exhibit a specific and uniform crystal shape, cubic, the Wulff equilibrium crystal structure. High-magnification images of individual domains further demonstrate that the CBs and SNPs are arranged in an alternating manner in the 3D cubic lattice (Fig. 3f,g). One can conclude that since CBs coordinate SNPs according to the symmetry of their faces, the space group of the formed lattice is strongly correlated with the point group of $\mathrm{CB}$ symmetry. In particular, the $\mathrm{CB}$ with point-group symmetry of $\mathrm{O}_{\mathrm{h}}$ directs the SNPs into a NaCl-type lattice with space-group symmetry of $F m \overline{3} m$, which is a subgroup of $O_{\mathrm{h}}$.

Effects of particle sizes and DNA shells. The DNA interactions play a central role in realizing the assembly of dissimilarly shaped CBs and SNPs. This strong interaction efficiently prevents phase segregation of the two components, which would otherwise occur because of shape mismatch. We further study the effects of DNA structure and particle size regimes on the formation of ordered assemblies of CBs and SNPs. First, we alter the DNA design (18S), which facilitated a superlattice formation, by making it more rigid. In this new design (DNA system denoted as 18R), the internal spacer region is an eight base-pair duplex. For the same 46-nm SNP/46-nm CB particle system previously described, the more rigid $18 R$ DNA linkages gave rise to only short-range order, even after annealing (refer to SAXS and SEM images, Fig. 4a-d). This suggests that using flexible DNA (18S) is essential for accommodating DNA bonds between the flat surface of $\mathrm{CB}$ and
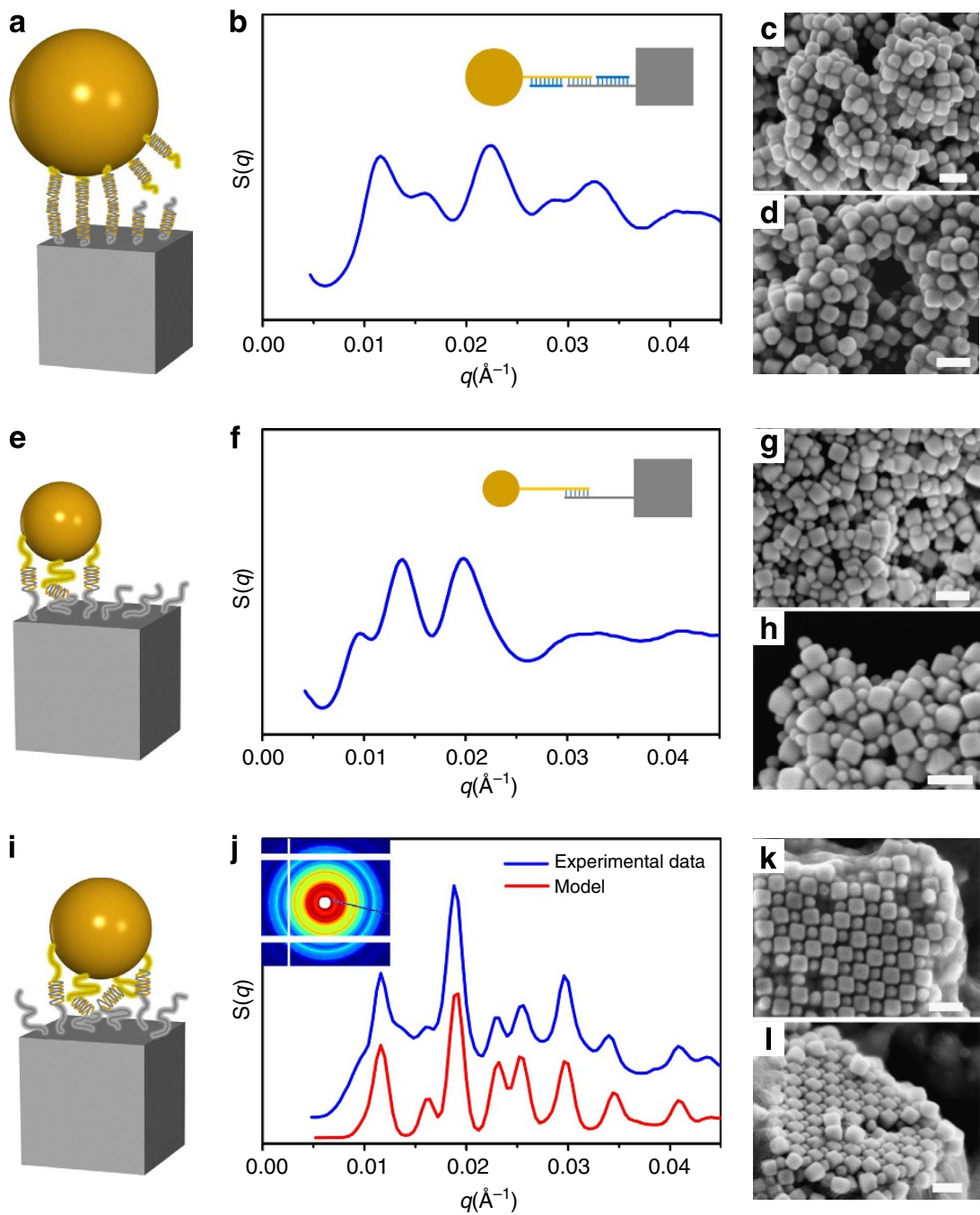

Figure 4 | Effects of DNA rigidity and sphere size on the structure of cube-sphere binary assembly. (a) Schematic of a 46-nm SNP/46-nm CB pair linked by rigid DNA system 18R. (b) SAXS extracted structure factors S(q) and (inset) DNA-NP schematic (18R). (c,d) SEM image of 46-nm SNP/46-nm CB assemblies for rigid DNA system 18R. (e) Schematic of a 27-nm SNP/46-nm CB pair linked by flexible DNA system 18S. (f) SAXS data and (inset) DNA-NP schematic (18S). (g,h) Corresponding SEM. (i) Schematic of a 38-nm SNP/46-nm CB pair linked by flexible DNA system 18S. (j) SAXS data with experimental (blue) and modelled (red) structure factors, S(q), and (inset) scattering image, which crystallizes into a NaCl-type lattice. (k,I) Corresponding SEM images. Scale bars, $100 \mathrm{~nm}$. 
the curved surface of SNP. Furthermore, the polymeric nature of flexible DNA spacer strands might help masking some size disparity between $\mathrm{CB}$ and SNP. To examine this size-mismatch tolerance, we probed the assemblies of 46-nm CBs with two types of smaller size SNPs (while maintaining the number ratio of 1:1), 27 and $38 \mathrm{~nm}$, respectively, using $18 S$ DNA. Size mismatches, $\Delta$, for 27 and $38 \mathrm{~nm}$ SNPs, are accordingly, $\sim 41 \%$ and $\sim 17 \%(\Delta$ is defined as $\left.\left(L_{\mathrm{CB}}-D_{\mathrm{SNP}}\right) / L_{\mathrm{CB}}\right)$. We observed the formation of only disordered morphologies for 27-nm SNPs (Fig. 4e-h), even though the same CB particles assembled with 38-nm SNPs formed a well-defined NaCl-type superlattice (Fig. 4i-l). SAXS pattern in Fig. 4j indicates about 12 sharp diffraction peaks and long-range order (correlation length $\sim 0.4 \mu \mathrm{m}$ ), demonstrating a good crystalline quality comparable to that of the size-matched system (46-nm SNPs/46-nm CBs, Fig. 3b). For this lattice, we obtained $a=108.3 \mathrm{~nm}$ and $d=12.2 \mathrm{~nm}$; the shorter surface-tosurface distance might indicate a moderate DNA chain compression in order to compensate for the size disparity of $\mathrm{CB}$ and SNP. The crystalline morphology of dry 38-nm SNP/46-nm CB sample was further visualized by SEM images (Fig. 4f,g). The top layers of the assembled superlattice show a well-preserved positional and orientational order of CBs, with each relatively small spherical particle is trapped inside a square space formed by four nearest neighbour CBs. The observed tolerance of ordered lattice for particle-size mismatch was also observed in systems with longer DNA strand and flexible spacer (Fig. 5a-c). DNA system 30 S consists of complementary 30-base DNAs, with each containing an 8-base outer recognition region and a 22-base internal spacer region. As shown in Figure 5a,, a large-scale organization with long-range order was observed for the 38-nm SNP/46-nm $\mathrm{CB}$ assemblies linked by $30 \mathrm{~S}$ DNA. Single-domain grains with up to micron-scale sizes have a cubic shape and relatively flat facets (Fig. 5b). CBs $(46 \mathrm{~nm})$ exhibit a remarkable orientational correlation and even enable isolating 38-nm SNPs on the top of layers (Fig. 5c).

We further investigated how the DNA structure and SNP size affect the shape-directed $\mathrm{CB}$ coordination of spheres, which is signified by the structure of $3 \mathrm{D}$ assembly. In Fig. 5d, we summarize the phase diagram with leading parameter being the DNA design and the SNP/CB size ratio for all the studied binary systems with 46-nm CB size. Several important conclusions can be drawn about the assembly of the presented heterogeneously shaped nanoparticles: (i) DNA structure and SNP size control assembly morphology, which varies from disordered aggregates to ordered lattices; (ii) flexible DNA motifs are essential for achieving crystalline organization (Supplementary Fig. 5), whereas the size match between dissimilar nano-objects is favourable but not strictly requisite; (iii) flexible DNA bridges are beneficial to the crystallization of size-mismatch SNP/CB assemblies (Figs $4 \mathrm{i}-\mathrm{l}$ and $5 \mathrm{a}-\mathrm{c}$ and Supplementary Fig. 6); (iv) DNA with excessively long flexible motifs results in short-range ordering even for size-matched particles (80S in Supplementary Fig. 5). The DNA shells comprising long and flexible chains mask the geometry; thus, the necessary plasticity of the interparticle connections can, if overemphasized, destroy the directional interactions provided by CBs, resulting in disordered assemblies. Furthermore, we observed that asymmetric shells, wherein the $\mathrm{CBs}$ and spheres were functionalized with DNA of different lengths while preserving total linker length within the crystalline zone (Fig. 5d), can induce yet more complex behaviour because of interplay of directional binding and polymeric effects (Supplementary Fig. 7).

The curved surfaces of SNPs restrict the number of DNA hybridizations that can occur without chain deformation, in comparison with interactions between flat surfaces. Indeed, DNA chains bridging the flat and curved surfaces can only maximize the number of bridges if some chains are stretched or compressed. For the size-matched NP assemblies (46-nm SNPs/ 46-nm CBs), the degree of DNA chain deformation, which depends on DNA structure (from $18 R$ to $18 S, 30 S, 80 S$ ), can be correlated with the state behaviour (Fig. 5d). In Fig. 5e, we compared the surface-to-surface distances, $D_{\text {ss }}$, in binary CB/SNP systems. We estimated $D_{\text {ss }}$ as the sum of DNA shell thicknesses on two types of surfaces, $T_{\text {DNA }}$ shell_cube $+T_{\text {DNA }}$ shell_sphere (Supplementary Note 4 ). We found that the observed $D_{\text {ss }}$ for the crystalline systems (18S and $30 S$ ) agrees well with our models, whereas the disordered systems (18R and $80 S)$ exhibit larger deviations. This suggests that DNA linkers that are too rigid or too long experience severe deviations from their equilibrium conformations, with a correspondingly greater energy penalty. Suitable DNA designs can properly balance the compression and stretching of DNA polymer chains confined between the differently curved surfaces of CB and SNP; thereby enabling the formation of well-defined crystalline nanoparticle assemblies.

The observed influence of the size mismatch (between SNP and CB linked by $18 S$ DNA) on lattice order can be understood by considering the details of interactions between the $\mathrm{CB}$ and SNP surfaces. The binding of SNPs in the middle of a CB's facet is an important factor for the order development, since it allows 'fixing' the subsequent attachments in a symmetric manner. SNP positioning in the middle of a $\mathrm{CB}$ face is favourable when $\Delta$ is small. Indeed, the adhesive energy between SNP and $\mathrm{CB}$ is proportional to the number of DNA chains hybridized between them, which can be approximated as the projected area of the $\mathrm{SNP}$ on the square face of the $\mathrm{CB}^{43}$. If the SNP diameter is smaller than the CB's edge length, then the SNP placement becomes ill-defined. In this case, the adhesive energy (approximately proportional to the number of DNA bridges) is equivalently maximized over a broad range of SNP positions on the $\mathrm{CB}$ face. In contrast, if the SNP and $\mathrm{CB}$ are comparable in size, the placement in the middle is uniquely favourable because of maximized adhesion (free energy minimized). This difference between a large free energy plateau and a well-localized minimum is essential for defining the particle position on the CB face. We calculated the attraction energy as function of the SNP displacement, $d_{\text {dis }}$, from the CB central axis crossing the CB's face centre (Fig. 5f and Supplementary Note 5). Decreasing the SNP's diameter results in an increase in the attractive potential plateau. The attraction potential curves between SNP and CB are schematically visualized by overlapping with $\mathrm{CB}$ shapes, as shown by the in-square red and black lines in Fig. 5f,g.

Figure $5 \mathrm{~g}$ illustrates the influence of this effect on lattice order. For matching sizes of SNP and CB, the SNPs are constrained into a very narrow equilibrium position on the CB's face, which promotes the alignment of CBs and SNPs over long ranges. In contrast, spread in the position of the small SNP on the face results in a random attachment of subsequent $\mathrm{CBs}$ and, consequently, in lattice disorder. However, as discussed above, a larger degree of mismatch can be compensated by longer chains of single-stranded DNAs (that is, DNA system 30S).

Assembly of octahedron and sphere nanoparticles. We further studied the assembly of heterogeneously shaped particles by choosing octahedron (OC) as anisotropic particle to assemble with spheres (Fig. 6a). The $\mathrm{OC}$ is a dual pair of the $\mathrm{CB}$, and belongs to the same symmetry group, but exposes eight triangular faces. We selected Au OCs and SNPs of $\sim 46 \mathrm{~nm}$ in the edge length and diameter, respectively. OCs and SNPs were functionalized with two types of complementary 50-base single-strand DNAs, $50 \mathrm{~A}$ and $50 \mathrm{~A}^{\prime}$, respectively (Fig. 6a and see Supplementary Fig. 1 and Supplementary Table 1), with each type of DNA 


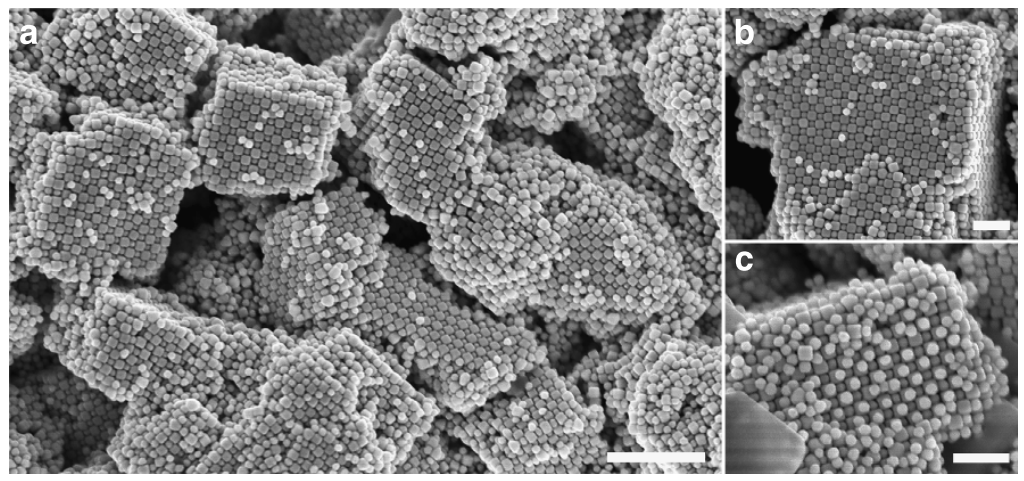

d

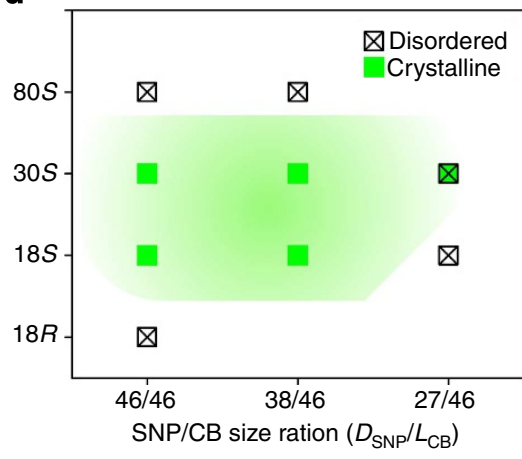

$\mathbf{f}$

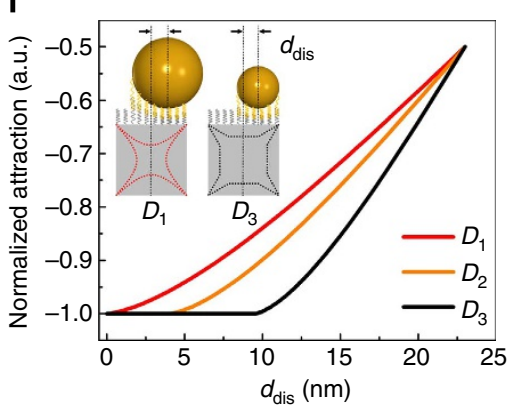

e

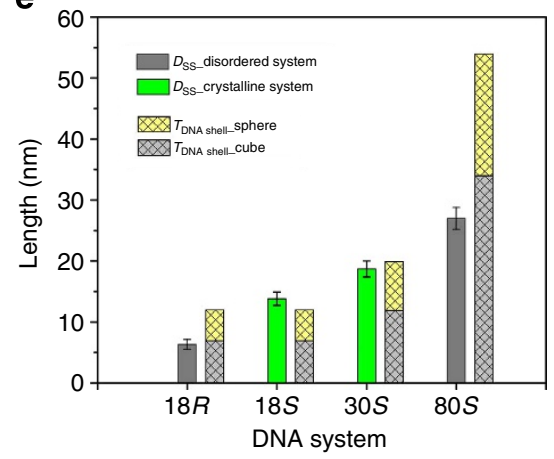

g
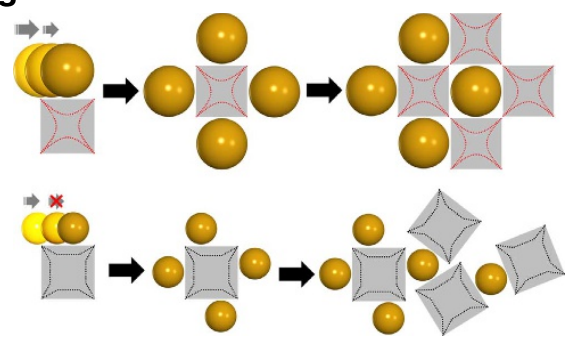

Figure 5 | Synergetic effects of DNA shell and particle size mismatch on the structure of cube-sphere binary assembly. (a) Low- and (b,c) highmagnification SEM images of binary superlattices formed by 38-nm SNPs/46-nm CBs with 30S DNA (left scale bar, $500 \mathrm{~nm}$; right scale bar, $200 \mathrm{~nm}$ ). (d) State diagram for all the studied binary systems of cubes, spheres and DNA designs. Leading parameters are the DNA length and flexibility, and the $\mathrm{SNP} / \mathrm{CB}$ size ratio (CB is $46 \mathrm{~nm}$ in edge length), as discussed in the text. (e) Comparison between experimental (from SAXS data) and calculated (see Supplementary Note 4 ) values of the nearest-neighbour interparticle surface-to-surface distances, $D_{\mathrm{ss}}$, for 46 -nm SNP/46-nm CB binary assembly (error bars correspond to one s.d. for repeated experiments). The calculated values account for the sum of DNA shell thicknesses on the cube and sphere

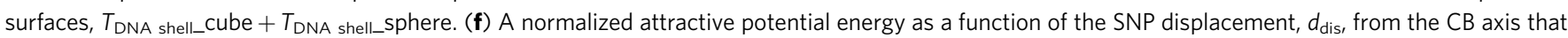
crosses the centre of the square face for SNPs with diameters of $46 \mathrm{~nm}$ (red line $D_{1}$ ), $38 \mathrm{~nm}$ (orange line $D_{2}$ ) and $27 \mathrm{~nm}$ (black line $D_{3}$ ), respectively, and schematics of SNP/CB pair for calculations (inset, left for $D_{1}$ and right for $D_{3}$ ). (g) Assembly of similarly sized and dissimilar CB and SNP. The mechanism for order propagation from the cluster formation of similar-sized cubes and spheres (top), and disorder (bottom) is illustrated, see the text for details.

containing an eight-base outer recognition region. The OC via its triangular facets provides eightfold coordination for SNPs. The SEM imaging indeed shows that OCs effectively coordinate spheres initially to some, and then to all, of the eight faces (Fig. 6b). Analogous to the CB's case, clusters formed by coordinating spheres around OCs merge into larger aggregates, in which OCs and SNPs alternate (Fig. $6 \mathrm{~b}$ and Supplementary Fig. 8).

The in situ SAXS measurements (Fig. 6c) demonstrate that the OCs and SNPs form a well-defined CsCl-type lattice, in which the OCs retain their orientational order, as evident from the experimental and modelled structure factors (blue and red curves, respectively, in Fig. 6d), which are in a good agreement (Supplementary Note 2). This bcc type phase ( $a$ of $\sim 82 \mathrm{~nm}$ and domain size of $\sim 0.4 \mu \mathrm{m}$ ) with a two-component basis can also be viewed as two interpenetrating simple cubic $(s c)$ lattices, wherein OCs and SNPs define the lattice sites of each sublattice (Fig. 6e). The $q_{1}$ position in the SAXS data corresponds to the $\{110\}$ crystalline planes in a CsCl-type lattice (Fig. 6e and Supplementary Note 3). In this lattice, eight SNPs surround each OC, and eight OCs surround each SNP, that is, the coordination number is 8 , which is reminiscent of perovskite crystals. SEM provides additional information about the arrangement of OCs and SNPs (Fig. 6f-h and Supplementary Fig. 9). Square domains and alternating shapes can be distinguished in the lowmagnification large-area image (Supplementary Fig. 9a). Highmagnification images confirm that the SNPs are attached to the triangular facets of OC; the alternating arrangement of OCs and $\mathrm{SNPs}$ in a CsCl-type lattice is clearly observed (Fig. 6f-h and Supplementary Fig. 9b-e). 
a

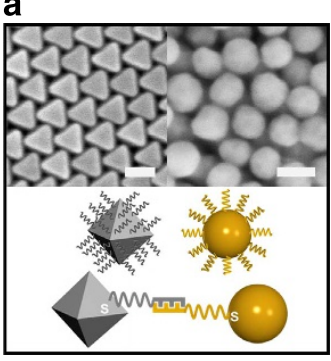

c
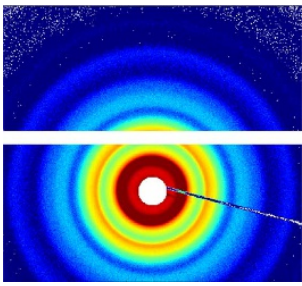

e

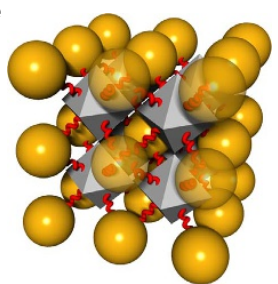

f

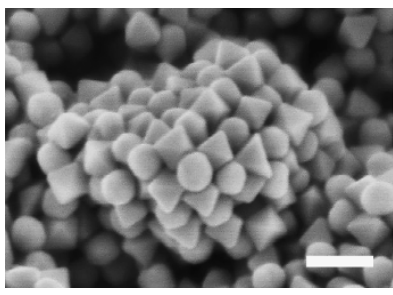

b

d
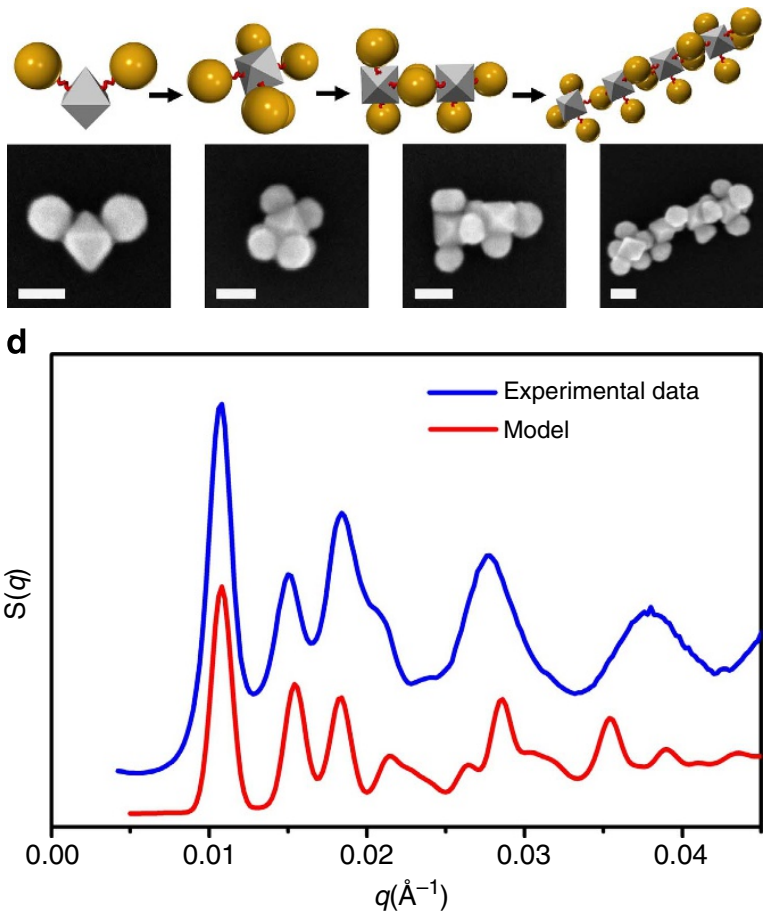

h

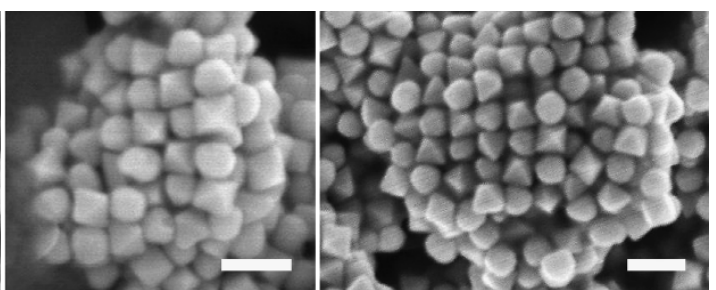

Figure 6 | Octahedron-sphere nanoparticle assemblies. (a; Top) SEM images of (from left to right) OCs (46 nm in edge length) and SNPs (46 nm in diameter); (bottom) schematic of DNA functionalization and assembly of OC and SNP. (b; Top) Schematic and (bottom) SEM images illustrating the directing role of octahedral blocks in the formation of clusters, and their merging into larger-scale structures (from left to right). Scale bars, $50 \mathrm{~nm}$. (c) SAXS image and (d) data with experimental (blue) and modelled (red) structure factors, $S(q)$. (e) The corresponding structure of formed CsCl-type superlattice with space group Pm $\overline{3} m$ symmetry (left bottom) for 46-nm SNP/46-nm OC assemblies. (f-h) SEM images of SNP/OC-assembled superlattice fragments (scale bar, $100 \mathrm{~nm}$ ).

\section{Discussion}

Notably, these results demonstrate that, despite having the same $O_{\mathrm{h}}$ point-group symmetry as $\mathrm{CBs}$, the OCs, due to their facet arrangement, direct SNPs into a different lattice. The OCs yield CsCl-like lattices $(b c c)$, whereas the CBs produce NaCl-like $(f c c)$ crystals. Both $F m \overline{3} m(\mathrm{NaCl})$ and $P m \overline{3} m(\mathrm{CsCl})$ are subgroups of the $O_{\mathrm{h}}$ point group, to which our polyhedral ANPs (CB and OC) belong, and in which they define a dual pair. However, as we observed, the formation of the specific lattice type from the group requires a particular symmetry of coordination of SNPs, and that is provided by the shape of the ANPs via its adhesive facets. To satisfy this seemingly simple rule, as we show, is not trivial, because of the effects of different local curvatures and interactions of dissimilarly shaped nano-objects. These effects play a crucial role in the formation of ordered assemblies. Hence, for optimized interaction in these lattices, the highest-order symmetry object, the sphere, is fully constrained by the low-order symmetry object, the anisotropic polyhedron.

In summary, we demonstrate that directional bonds provided by polyhedral nanoparticles can be used effectively for arranging isotropically interacting spherical nanoparticles into $3 \mathrm{D}$ superlattices. We uncover the primary requirements for the geometrical features of polyhedral blocks to be robustly translated into 3D crystalline order; namely, appropriate tuning of DNA interactions and particle size regimes. Moreover, the origin of global lattice symmetry can be traced back to the geometry of the anisotropic blocks. The softness of particle coronas is crucial for accommodating mismatched shapes in the lattice, however, excessive flexibility results in disordered states. Furthermore, the relative sizes of dissimilar particles are imperative; longrange-ordered lattices only form when particles assemble so as to locally constrain positional order. The presented work should permit more broadly for the realization of designed heteroassemblies, using directional interactions of anisotropic nanoblock and DNA programmability, and it will stimulate fundamental studies on the relationship between building block geometry and global crystallographic symmetry. The role of the particle's soft shell cannot be overstated. Corona softness is crucial to the formation of an accommodating interface between the differently shaped particles, thereby promoting ordered phases despite conflicting directional interactions. An extensive variety of nanoscale polyhedral objects have become available, 
such as tetrahedron, decahedron and icosahedron ${ }^{27,44-46 ;}$ simultaneously, DNA-coating approaches have been extended to many different nanomaterial types $3,47,48$. The demonstrated approach will thus open novel opportunities for the rational design of 3D nanostructured materials from a broad variety of functional nanocomponents.

\section{Methods}

Synthesis of polyhedral gold nanoparticles. Reagents: Gold (III) chloride trihydrate $\left(\mathrm{HAuCl}_{4} \cdot 3 \mathrm{H}_{2} \mathrm{O}, 99.9+\%\right)$, sodium borohydrate $\left(\mathrm{NaBH}_{4}, 99.99 \%\right)$, L-ascorbic acid (AA, $99+\%$ ), cetyltrimethylammonium bromide (CTAB, 99.9\%) and cetylpyridinium chloride (CPC, 99\%) were purchased from Sigma-Adrich and used without further purification. Milli-Q water with a resistivity greater than $18.0 \mathrm{M} \Omega \mathrm{cm}$ was used in the preparation of aqueous solutions. Synthesis of $\mathrm{Au}$ nano-octahedral seeds: The Au nano-octahedral seeds were prepared using a modified two-step procedure ${ }^{49}$. First, $3-\mathrm{nm}$ Au seeds were prepared by quickly injecting $0.60 \mathrm{ml}$ of ice-cold, freshly prepared $\mathrm{NaBH}_{4}(10 \mathrm{mM})$ into a rapidly stirred mixture of $\mathrm{HAuCl}_{4}(10 \mathrm{mM}, 0.25 \mathrm{ml})$ and CTAB $(0.1 \mathrm{M}, 9.75 \mathrm{ml})$. The seed solution was stirred for $2 \mathrm{~min}$ and then left undisturbed at $25^{\circ} \mathrm{C}$ for $3 \mathrm{~h}$ to ensure complete decomposition of $\mathrm{NaBH}_{4}$ remaining in the solution. The seed solution was diluted 100 times with $0.1 \mathrm{M} \mathrm{CTAB}$. For the synthesis of octahedral Au seeds, $4 \mathrm{ml}$ of aqueous $\mathrm{HAuCl}_{4}$ solution $(0.3 \mathrm{mM}), 24 \mathrm{ml}$ of aqueous CTAB solution $(20 \mathrm{mM})$ and $1.8 \mathrm{ml}$ of aqueous AA solution $(100 \mathrm{mM})$ were mixed, followed by adding $360 \mu \mathrm{l}$ of the diluted seed solution under stirring. The mixture solution was then left to age at $25^{\circ} \mathrm{C}$ for $12 \mathrm{~h}$. Seed-mediated growth of polyhedral Au nanoparticles: In a typical synthesis of the Au nanocubes, $5 \mathrm{ml}$ of the above 12-h-aged seeds was first washed three times with $0.1 \mathrm{M} \mathrm{CTAB}$ solution using centrifugation (10,000 r.p.m., $10 \mathrm{~min}$ ) and finally condensed in $500 \mu \mathrm{l}$ of $16 \mathrm{mM} \mathrm{CTAB}$ solution; $100 \mu \mathrm{l}$ of $10 \mathrm{mM} \mathrm{HAuCl}_{4}$ solution, $400 \mu \mathrm{l}$ of freshly prepared $100 \mathrm{mM} \mathrm{AA}$ and $500 \mu \mathrm{l}$ of the condensed seed solution were added to $5 \mathrm{ml}$ of $16 \mathrm{mM} \mathrm{CTAB}$ solution at $32^{\circ} \mathrm{C}$ consecutively and thoroughly mixed after each addition. The reaction was then left undisturbed for $6 \mathrm{~h}$ and stopped by centrifugation (10,000 r.p.m., $10 \mathrm{~min})$. The obtained nanoparticle solution was washed twice with Milli-Q water and concentrated for further usages. In a typical synthesis of the Au OCs, $5 \mathrm{ml}$ of the above 12-h-aged seeds was first washed three times with $0.1 \mathrm{M} \mathrm{CPC}$ solution by centrifugation $(10,000$ r.p.m., $10 \mathrm{~min})$ and finally condensed in $500 \mu \mathrm{l}$ of $0.1 \mathrm{M} \mathrm{CPC}$ solution; $100 \mu \mathrm{l}$ of $10 \mathrm{mM} \mathrm{HAuCl}_{4}$ solution, $13 \mu \mathrm{l}$ of freshly prepared $100 \mathrm{mM} \mathrm{AA}$ and $500 \mu \mathrm{l}$ of the condensed seed solution were added to $5 \mathrm{ml}$ of $0.1 \mathrm{M} \mathrm{CPC}$ solution at $25^{\circ} \mathrm{C}$ consecutively and thoroughly mixed after each addition. The reaction was then left undisturbed for $6 \mathrm{~h}$ and stopped by centrifugation (10,000 r.p.m., $10 \mathrm{~min})$. The resultant nanoparticle solution was washed twice with Milli-Q water and concentrated for further usage. Average edge lengths of the synthesized $\mathrm{Au}$ nanocubes and nano-OCs are similar, being of $\sim 46 \mathrm{~nm}$. Au nanocubes have a size distribution s.d. of $\sim 2 \%$ and a yield of $\sim 96 \%$; Au nano-OCs have a size distribution s.d. of $\sim 3 \%$ and a yield of $\sim 95 \%$. The results were obtained from counting at least 200 nanoparticles for each sample.

Nanoparticle functionalization with DNA. Thiol-modified single-strand oligonucletides (see Supplementary Table 1 for sequences) were purchased from Integrated DNA Technologies Inc. with disulfide modification. Before nanoparticle DNA functionalization, the disulfide oligonucleotides were first reduced by dissolving the lyophilized samples $(100 \sim 300$ nmoles $)$ in $0.3 \mathrm{ml}$ of a $100-\mathrm{mM}$ dithiothreitol (DTT) solution in purified water or buffer. The reduced DNA was loaded on a freshly purified sephadex column (G-25, Amersham Bioscience) and eluted with $2.5 \mathrm{ml}$ of $10 \mathrm{mM}$ phosphate buffer $(\mathrm{pH}=7.4)$. The DNA was quantified using UV-vis analysis using the known extinction coefficient. Au nanoparticles were functionalized with ssDNA following a previously demonstrated method to achieve high DNA coverage ${ }^{32}$. Briefly, an aliquot of purified DNA solution was added to $1 \mathrm{ml}$ aliquot of Au nanoparticles $\left(\sim 3 \mathrm{OD}_{260}\right.$ of DNA for per $\mathrm{ml}$ of nanoparticle colloid). After allowing 1-3 h for thiolated DNAs to react with the gold surface, particle suspensions were brought to $0.01 \%$ sodium dodecyl sulfate (SDS) and $10 \mathrm{mM}$ sodium phosphate and allowed to sit for $1 \mathrm{~h}$. Following literature procedures, the colloidal nanoparticle solutions were then slowly treated with $\mathrm{NaCl}$ to allow for electrostatic screening between neighbouring DNA strands and denser surface coverage of oligonucleotides. Specifically, $\mathrm{NaCl}$ concentration of the solution was brought to $0.5 \mathrm{M}$ slowly by adding aliquots of $3 \mathrm{M} \mathrm{NaCl}$ eight times with 30-min interval for incubation. After reaching the final $\mathrm{NaCl}$ concentration, particles were allowed to sit overnight to achieve maximum DNA loading. To remove the excess, unbound DNA from the solution, the mixture was centrifuged, the supernatant was removed and the pellet was resuspended in washing buffer $(0.01 \% \mathrm{SDS}+10 \mathrm{mM}$ phosphate buffer, $\mathrm{pH}=7.4)$. This process was repeated three times. The final resuspension typically occurred in $50-100 \mu \mathrm{l}$ to allow for a concentrated solution of particles in $0.2 \mathrm{M}$ PBS buffer $(0.2 \mathrm{M} \mathrm{NaCl}+10 \mathrm{mM}$ phosphate buffer, $\mathrm{pH}=7.4)$ for further assemblies. Concentration of nanoparticles was quantified using the absorbance value at the surface plasmon resonance (SPR) maximum in UV-vis absorption spectra. A molar extinction coefficient of $3.5 \times 10^{10} \mathrm{M}^{-1} \mathrm{~cm}^{-1}$ at $546 \mathrm{~nm}$ SPR peak is used for the nanocubes with $\sim 46 \mathrm{~nm}$ edge. A molar extinction coefficient of $2.5 \times 10^{10} \mathrm{M}^{-1} \mathrm{~cm}^{-1}$ at the
$564 \mathrm{~nm}$ SPR peak is used for the nano-OCs with $\sim 46 \mathrm{~nm}$ edge. For the spherical $\mathrm{Au}$ nanoparticles purchased from Nanopartz Inc., molar extinction coefficients of $10.5 \times 10^{9} \mathrm{M}^{-1} \mathrm{~cm}^{-1}$ at the $532 \mathrm{~nm}$ peak, $5.93 \times 10^{9} \mathrm{M}^{-1} \mathrm{~cm}^{-1}$ at $529 \mathrm{~nm}$ peak and $3.6 \times 10^{9} \mathrm{M}^{-1} \mathrm{~cm}^{-1}$ at $527 \mathrm{~nm}$ are used for the nanospheres with the diameters of 46,38 and $27 \mathrm{~nm}$, respectively.

Assembly and crystallization of nanoparticle superlattices. The assembly was obtained by combining equal molar amounts of type-A and type- $\mathrm{A}^{\prime}$ DNA-functionalized Au nanoparticles, and the particles were allowed to aggregate at room temperature. The samples were then annealed at a temperature $\sim 1-2^{\circ} \mathrm{C}$ below the melting temperature of the assembled particles for a period of several hours, depending on the sample. The annealed samples were transferred with buffer to a quartz capillary ( $1.0 \mathrm{~mm}$ diameter), and sealed with wax for SAXS measurements.

Characterization of nanoparticles and assemblies. The morphology of nanoparticles and the ex situ structure of assemblies were characterized using electron microscopy. Synchrotron-based SAXS (NSLS X9) was used to probe the in situ structure of particle assemblies. UV-Visible Spectrophotometry (UV-vis): UV-vis spectra were recorded on a Perkin-Elmer Lambda 35 spectrometer $(200-700 \mathrm{~nm})$ Melting analysis was performed in conjunction with a Perkin-Elmer PTP-1 Peltier Temperature Programmer between 20 and $75^{\circ} \mathrm{C}$ with a temperature ramp of $1^{\circ} \mathrm{C} \mathrm{min}^{-1}$ while stirring, in a $10 \mathrm{mM}$ phosphate buffer, $0.2 \mathrm{M} \mathrm{NaCl}, \mathrm{pH}=7.4$, buffer solution. SEM: SEM experiments were carried out on Hitachi S-4800 Scanning Electron Microscopy with typical $1 \mathrm{kV}$ voltage and $10 \mu \mathrm{A}$ emission current. The sample was prepared by drop-casting an aqueous nanoparticle solution on a cleaned silicon substrate. TE): TEM images were collected on a JEOL-1400 microscope operated at $120 \mathrm{kV}$. The samples were prepared by drop-casting an aqueous nanoparticle solution on a carbon-coated copper grid. Small-angle X-ray scattering (SAXS): SAXS experiments were performed in situ at the X9 beamline at the National Synchrotron Light Source. The scattering data were collected with a Dectris Pilatus $1 \mathrm{M}$ pixel-array detector and converted to $1 \mathrm{D}$ scattering intensity versus wave vector transfer, $q=(4 \pi / \lambda) \sin (\theta / 2)$, where $\lambda=0.9184 \AA$, and $\theta$, are the wavelength of incident X-ray beam and the full scattering angle, respectively. The data are presented as the structure factor $S(q)$, which was calculated as $I_{\mathrm{a}}(q) / I_{\mathrm{p}}(q)$, where $I_{\mathrm{a}}(q)$ and $I_{\mathrm{p}}(q)$ are background-corrected 1D scattering intensities extracted by angular averaging of detector images for a system under consideration, and the corresponding unaggregated gold particles, respectively. The peak positions in $S(q)$ are determined by fitting to a Lorentzian function.

Modelling of SAXS profiles and DNA structure. To simulate powder SAXS profiles, we used our recently published scattering formalism, which simulates powder SAXS profiles for lattices of particles with arbitrary shape ${ }^{42}$. This formalism accounts for particle size, particle shape and particle orientation within the unit cell. We also explicitly included disorder: particle size polydispersity, lattice disorder (Debye-Waller factor) and average grain size. We used a polyelectrolyteblob model and a Daoud-Cotton (DC) blob model to calculate the tethered DNA thickness on a flat surface (for cube) and a curved surface (for sphere), respectively. All SAXS and DNA modelling details are provided in the Supplementary Note 2.

SEM and layer-by-layer sample preparation. The sample deposited on a cleaned silicon substrate was measured using a Hitachi S-4800 Scanning Electron Microscope with typical $1-\mathrm{kV}$ voltage and $10-\mu \mathrm{A}$ emission current. A standard polyelectrolyte-assisted layer-by-layer (LBL) method was applied to prepare the diluted nanoparticle-assembled clusters for SEM characterization ${ }^{50}$. Silicon wafers were used as substrates for SEM characterization. The substrates were sonicated for $10 \mathrm{~min}$ in water and then in ethanol, subsequently thoroughly cleaned using piranha solution $\left(\mathrm{H}_{2} \mathrm{SO}_{4}: \mathrm{H}_{2} \mathrm{O}_{2}=7: 3\right)$, rinsed with deionized water and dried under an air stream. The wafers were stored in water before being use. The wafers were first immersed in an aqueous solution of positively charged poly (diallyldimethylammonium chloride) PDDA ( $\mathrm{Mw}=200,000,1 \mathrm{mg} \mathrm{ml}^{-1}$ in $0.5 \mathrm{M}$ $\mathrm{NaCl}$ aqueous solution) for $20 \mathrm{~min}$, and then in an aqueous solution of the polyanion poly(acrylic acid, sodium salt) PAA (Mw $=15,000,1 \mathrm{mg} \mathrm{ml}^{-1}$ in $0.5 \mathrm{M}$ $\mathrm{NaCl}$ aqueous solution) for $10 \mathrm{~min}$ and finally in PDDA solution for $10 \mathrm{~min}$. At this stage, the wafers are positively charged, favouring the electrostatic interaction with negatively charged DNA in the assembled aggregates. To obtain a monolayer of nanoparticle-assembled clusters, the pretreated wafers were immersed into the corresponding solution with diluted aggregates and kept for a suitable period time After enough absorption, the substrates were rinsed with deionized water and dried under an air stream for further SEM characterization.

Quantification of DNA grafting. A fluorescence-based method was used to determine the number of DNA loaded on particle surfaces ${ }^{51}$. First, the DNA was chemically displaced from the nanoparticle surface using DTT. The displacement was initiated by adding equal volumes of DNA-functionalized nanoparticles and 1.0 M DTT in 0.1 M PBS, $\mathrm{pH}=7.4$. The oligonucleotides were released into solution during overnight incubation, and the particle precipitate was removed by centrifugation. The concentrations of oligonucleotide in solution were determined using fluorescence spectroscopy. During the fluorescence measurement, the 
fluorophore was excited at $450 \mathrm{~nm}$ and the emission was collected from 520 to $640 \mathrm{~nm}$.

\section{References}

1. Kuzyk, A. et al. DNA-based self-assembly of chiral plasmonic nanostructures with tailored optical response. Nature 483, 311-314 (2012).

2. Henry, E. et al. Crystallization of fluorescent quantum dots within a threedimensional bio-organic template of actin filaments and lipid membranes. Nano. Lett. 11, 5443-5448 (2011).

3. Zhang, Y. G., Lu, F., Yager, K. G., van der Lelie, D. \& Gang, O. A general strategy for the DNA-mediated self-assembly of functional nanoparticles into heterogeneous systems. Nat. Nanotechnol. 8, 865-872 (2013).

4. Nykypanchuk, D., Maye, M. M., van der Lelie, D. \& Gang, O. DNA-guided crystallization of colloidal nanoparticles. Nature 451, 549-552 (2008).

5. Macfarlane, R. J. et al. Nanoparticle superlattice engineering with DNA. Science 334, 204-208 (2011).

6. Xiong, H. M., van der Lelie, D. \& Gang, O. Phase behavior of nanoparticles assembled by DNA linkers. Phys. Rev. Lett. 102 (2009).

7. Casey, M. T. et al. Driving diffusionless transformations in colloidal crystals using DNA handshaking. Nat. Commun. 3, 1209 (2012).

8. Varrato, F. et al. Arrested demixing opens route to bigels. Proc. Natl Acad. Sci. USA 109, 19155-19160 (2012).

9. Xia, Y. S. et al. Self-assembly of self-limiting monodisperse supraparticles from polydisperse nanoparticles. Nat. Nanotechnol. 6, 580-587 (2011).

10. Leunissen, M. E. et al. Ionic colloidal crystals of oppositely charged particles. Nature 437, 235-240 (2005).

11. Shevchenko, E. V., Talapin, D. V., Kotov, N. A., O'Brien, S. \& Murray, C. B. Structural diversity in binary nanoparticle superlattices. Nature 439, 55-59 (2006).

12. Chen, Q. et al. Supracolloidal reaction kinetics of janus spheres. Science 331, 199-202 (2011).

13. Chen, Q., Bae, S. C. \& Granick, S. Directed self-assembly of a colloidal kagome lattice. Nature 469, 381-384 (2011).

14. Romano, F., Sanz, E. \& Sciortino, F. Phase diagram of a tetrahedral patchy particle model for different interaction ranges. J. Chem. Phys. 132, 184501 (2010).

15. Pawar, A. B. \& Kretzschmar, I. Fabrication, assembly, and application of patchy particles. Macromol. Rapid Commun. 31, 150-168 (2010).

16. Romano, F. \& Sciortino, F. Colloidal self-assembly patchy from the bottom up. Nat. Mater. 10, 171-173 (2011).

17. Wang, Y. F. et al. Colloids with valence and specific directional bonding. Nature 491, 51-U61 (2012).

18. Yi, G. R., Pine, D. J. \& Sacanna, S. Recent progress on patchy colloids and their self-assembly. J. Phys. Condens. Matter 25, 193101 (2013).

19. Russo, J., Tartaglia, P. \& Sciortino, F. Association of limited valence patchy particles in two dimensions. Soft Matter 6, 4229-4236 (2010).

20. Tavares, J. M., Almarza, N. G. \& da Gama, M. M. T. Three-dimensional patchy lattice model: Ring formation and phase separation. J. Chem. Phys. 140, 044905 (2014).

21. Maye, M. M., Nykypanchuk, D., Cuisinier, M., van der Lelie, D. \& Gang, O. Stepwise surface encoding for high-throughput assembly of nanoclusters. Nat. Mater. 8, 388-391 (2009).

22. Kim, J. W., Kim, J. H. \& Deaton, R. DNA-Linked Nanoparticle Building Blocks for Programmable Matter. Angew. Chem.-Int. Ed. 50, 9185-9190 (2011).

23. Zhang, C. et al. DNA nanocages swallow gold nanoparticles (AuNPs) to form AuNP@DNA cage core-shell structures. ACS Nano 8, 1130-1135 (2014).

24. Adams, M., Dogic, Z., Keller, S. L. \& Fraden, S. Entropically driven microphase transitions in mixtures of colloidal rods and spheres. Nature 393, 349-352 (1998).

25. Ye, X. C. et al. Shape alloys of nanorods and nanospheres from self-assembly. Nano. Lett. 13, 4980-4988 (2013).

26. Khadilkar, M. R., Agarwal, U. \& Escobedo, F. A. Phase behavior of binary mixtures of hard convex polyhedra. Soft Matter 9, 11557-11567 (2013).

27. Xia, Y. N., Xia, X. H., Wang, Y. \& Xie, S. F. Shape-controlled synthesis of metal nanocrystals. MRS Bull. 38, 335-344 (2013).

28. Damasceno, P. F., Engel, M. \& Glotzer, S. C. Predictive self-assembly of polyhedra into complex structures. Science 337, 453-457 (2012).

29. van Anders, G., Ahmed, N. K., Smith, R., Engel, M. \& Glotzer, S. C. Entropically patchy particles: engineering valence through shape entropy. ACS Nano 8, 931-940 (2014).

30. Henzie, J., Grunwald, M., Widmer-Cooper, A., Geissler, P. L. \& Yang, P. D. Self-assembly of uniform polyhedral silver nanocrystals into densest packings and exotic superlattices. Nat. Mater. 11, 131-137 (2012).

31. Torquato, S. \& Jiao, Y. Dense packings of the Platonic and Archimedean solids. Nature 460, 876-U109 (2009).

32. Jones, M. R. et al. DNA-nanoparticle superlattices formed from anisotropic building blocks. Nat. Mater. 9, 913-917 (2010).
33. Alvarez-Puebla, R. A. et al. Gold nanorods 3D-supercrystals as surface enhanced Raman scattering spectroscopy substrates for the rapid detection of scrambled prions. Proc. Natl Acad. Sci. USA 108, 8157-8161 (2011).

34. Ye, X. C. et al. Competition of shape and interaction patchiness for selfassembling nanoplates. Nat. Chem. 5, 466-473 (2013).

35. Rossi, L. et al. Cubic crystals from cubic colloids. Soft Matter 7, 4139-4142 (2011).

36. Agarwal, U. \& Escobedo, F. A. Mesophase behaviour of polyhedral particles. Nat. Mater. 10, 230-235 (2011).

37. Sacanna, S., Irvine, W. T. M., Chaikin, P. M. \& Pine, D. J. Lock and key colloids Nature 464, 575-578 (2010)

38. Paik, T. \& Murray, C. B. Shape-directed binary assembly of anisotropic nanoplates: a nanocrystal puzzle with shape-complementary building blocks. Nano. Lett. 13, 2952-2956 (2013).

39. Millan, J. A., Ortiz, D., van Anders, G. \& Glotzer, S. C. Self-assembly of Archimedean tilings with enthalpically and entropically patchy polygons. ACS Nano 8, 2918-2928 (2014).

40. Khadilkar, M. R. \& Escobedo, F. A. Heuristic rule for binary superlattice coassembly: mixed plastic mesophases of hard polyhedral nanoparticles. Phys. Rev. Lett. 113 (2014).

41. Knorowski, C. \& Travesset, A. Self-assembly and crystallization of hairy (f-Star) and DNA-grafted nanocubes. J. Am. Chem. Soc. 136, 653-659 (2014).

42. Yager, K. G., Zhang, Y. G., Lu, F. \& Gang, O. Periodic lattices of arbitrary nanoobjects: modeling and applications for self-assembled systems. J. Appl. Crystallogr. 47, 118-129 (2014).

43. Nykypanchuk, D., Maye, M. M., van der Lelie, D. \& Gang, O. DNA-based approach for interparticle interaction control. Langmuir 23, 6305-6314 (2007).

44. Lu, F. et al. Truncated ditetragonal gold prisms as nanofacet activators of catalytic platinum. J. Am. Chem. Soc. 133, 18074-18077 (2011).

45. Yang, M., Sun, K. \& Kotov, N. A. Formation and assembly-disassembly processes of $\mathrm{ZnO}$ hexagonal pyramids driven by dipolar and excluded volume interactions. J. Am. Chem. Soc. 132, 1860-1872 (2010).

46. Zheng, Y. Q. et al. Seed-mediated synthesis of gold tetrahedra in high purity and with tunable, well-controlled sizes. Chem. Asian J. 9, 2635-2640 (2014).

47. Zhang, C. et al. A general approach to DNA-programmable atom equivalents. Nat. Mater. 12, 741-746 (2013).

48. Lu, F. et al. Discrete nanocubes as plasmonic reporters of molecular chirality. Nano. Lett. 13, 3145-3151 (2013).

49. Fan, F. R. et al. Epitaxial growth of heterogeneous metal nanocrystals: from gold nano-octahedra to palladium and silver nanocubes. J. Am. Chem. Soc. 130, 6949-6951 (2008).

50. Vial, S., Pastoriza-Santos, I., Perez-Juste, J. \& Liz-Marzan, L. M. Plasmon coupling in layer-by-layer assembled gold nanorod films. Langmuir 23, 4606-4611 (2007).

51. Hurst, S. J., Lytton-Jean, A. K. R. \& Mirkin, C. A. Maximizing DNA loading on a range of gold nanoparticle sizes. Anal. Chem. 78, 8313-8318 (2006).

\section{Acknowledgements}

Research was carried out at the Center for Functional Nanomaterials, Brookhaven National Laboratory, supported by the US Department of Energy, Office of Basic Energy Sciences, under Contract No. DE-AC02-98CH10886.

\section{Author contributions}

F.L. and O.G. conceived the concept and designed the experiments. F.L. performed the experiments. F.L. and O.G. analysed the data. K.G.Y. performed SAXS modelling and analysis. Y.G.Z. contributed to the DNA modelling and SAXS analysis. H.L.X. contributed to TEM characterization and tomographic reconstructions. F.L. and O.G. wrote the paper. All authors discussed the results and commented on the manuscript.

\section{Additional information}

Supplementary Information accompanies this paper at http://www.nature.com/ naturecommunications

Competing financial interests: The authors declare no competing financial interests.

Reprints and permission information is available online at http://npg.nature.com/ reprintsandpermissions/

How to cite this article: Lu, F. et al. Superlattices assembled through shape-induced directional binding. Nat. Commun. 6:6912 doi: 10.1038/ncomms7912 (2015).

This work is licensed under a Creative Commons Attribution 4.0 International License. The images or other third party material in this article are included in the article's Creative Commons license, unless indicated otherwise in the credit line; if the material is not included under the Creative Commons license, users will need to obtain permission from the license holder to reproduce the material. To view a copy of this license, visit http://creativecommons.org/licenses/by/4.0/ 